Investigating the Survival Benefit of Combining Radiotherapy for Surgery Treated Locally Advanced Esophageal Squamous Cell Carcinoma Patients Aged 65 and Older

\title{
Huang, Shan
}

2019-11

Huang , S , Li , Y , Ma , H , Wang , W , Zheng , S , Ke , Y \& Li , F 2019 , ' Investigating the Survival Benefit of Combining Radiotherapy for Surgery Treated Locally Advanced Esophageal Squamous Cell Carcinoma Patients Aged 65 and Older ' , Journal of Gastrointestinal Surgery , vol. 23 , no. 11 , pp. 2111-2118 . https://doi.org/10.1007/s11605-019-04200-5

http://hdl.handle.net/10138/322089

https://doi.org/10.1007/s11605-019-04200-5

publishedVersion

Downloaded from Helda, University of Helsinki institutional repository.

This is an electronic reprint of the original article.

This reprint may differ from the original in pagination and typographic detail.

Please cite the original version. 


\title{
Investigating the Survival Benefit of Combining Radiotherapy for Surgery Treated Locally Advanced Esophageal Squamous Cell Carcinoma Patients Aged 65 and Older
}

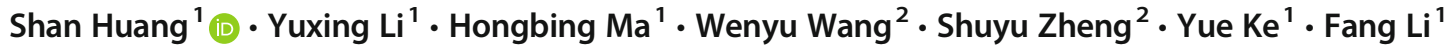

Received: 23 August 2018 / Accepted: 5 March 2019/Published online: 20 March 2019

(C) 2019 The Society for Surgery of the Alimentary Tract

\begin{abstract}
Purpose To evaluate the survival benefit of combining radiotherapy with surgery in locally advanced esophageal squamous cell carcinoma (ESCC) patients aged over 65.

Methods Using the SEER database, we selected patients age $\geq 65$ years that were diagnosed as locally advanced ESCC during 2004-2013. Cancer-specific survival (CSS) was examined using the Kaplan-Meier analysis and compared by the log-rank test. Univariable and multivariable Cox proportional hazard models were established to identify possible prognostic factors.

Results A total of 972 cases were included in the study. For surgical patients aged 65-79 years, 74 patients (32.9\%) were treated by surgery alone and 122 patients $(54.2 \%)$ had received additional neoadjuvant radiotherapy (NRT). NRT + surgery was associated with improved CSS comparing with surgery alone (HR, $0.58 ; 95 \% \mathrm{CI}, 0.39$ to $0.85 ; P=0.005$ ). In subgroup analysis, NRT was associated with improved CSS for patients aged 65-74 years (2-year CSS 56.6\% versus $39.6 \%, P=0.026$ ). No significant differences of progonosis was observed for different treatment groups in $75-79$ years patients $(P=0.972)$.

Conclusions In this SEER-based study, the addition of neoadjuvant radiotherapy before surgery was associated with improved CSS for locally advanced ESCC patients aged 65 to 74 years.
\end{abstract}

Keywords Esophageal squamous cell carcinoma $\cdot$ Aged 65 and older $\cdot$ Surgery $\cdot$ Radiotherapy $\cdot$ Survival

\section{Introduction}

Esophageal cancer (EC) is the ninth most common malignancy and ranks sixth in cancer deaths worldwide. ${ }^{1}$ Esophageal squamous cell carcinoma (ESCC) is the most common histological type in Asian contries. ${ }^{2}$ EC is also a disease for the elderly, with a median age of 67 years at diagnosis. ${ }^{3,4}$ The mortality rate of EC rises with increasing age. In 2010-2014,

Electronic supplementary material The online version of this article (https://doi.org/10.1007/s11605-019-04200-5) contains supplementary material, which is available to authorized users.

\section{Shan Huang}

huangshan2016@xjtu.edu.cn

1 Department of Radiation Oncology, Second Affiliated Hospital, Xi'an Jiaotong University, No.157, Xi Wu Road, Xi'an 710004, Shaanxi, China

2 Institute for Molecular Medicine Finland (FIMM), Helsinki Institute of Life Science, University of Helsinki, 00014 Helsinki, Finland the mortality rate of EC in the USA was $15.8 / 100,000$ for 65 69 years old, which was more than three times $(4.3 / 100,000)$ than patients aged $50-54$ years. ${ }^{3}$ The much worse mortalrity rate in elderly EC patients suggested the possible limitation of current treatment strategy.

Preoperative chemotherapy and neoadjuvant radiotherapy (NRT) followed by surgery has become the standard treatment for locally advanced esophageal cancer. The NEOCRTEC5010 trial compared the survival of patients receiving preoperative chemoradiotherapy + surgery to the survival of patients with surgery alone. ${ }^{5}$ They found that for patients with locally advanced ESCC, those who received additional preoperative chemoradiotherapy showed an increase of 33.6 months median overall survival. However, the FFCD9901 trial showed a threefold increased postoperative mortality in the patients treated by neoadjuvant chemoradiotherapy + surgery group than that of surgery alone. ${ }^{6}$

This raised the question of whether and when additional radiotherapy (RT) should be applied. In elderly patients in particular, the toxicity of chemotherapy and RT is quite predominant. For example, $38.2 \%$ patients aged $\geq 75$ years had 
grade 3 or 4 toxicity and nearly three quarters of patients required hospital visit and/or RT break. ${ }^{7}$ In the existing studies, very few elderly patients (aged over 65 years) were included. ${ }^{8}$ Whether the elderly surgery patients benefit from additional RT remains to be elucidated.

In this study, we analyzed the Surveillance, Epidemiology, and End Results (SEER) Program of the National Cancer Institute (NCI) database to evaluate the survival impact of additional RT for locally advanced ESCC surgery patients aged 65 and older.

\section{Materials and Methods}

\section{Data Source}

SEER collects cancer incidence and survival data from population-based cancer registries covering approximately $34.6 \%$ of the US population. The SEER Program collects data on patients demographics, stage of cancer at the time of diagnosis, and patient survival.

This study used SEER database data from 1973 to 2013 ("Incidence-SEER 18 Regs Research Data + Hurricane Katrina Impacted Louisiana Cases, Nov 2015 Sub (19732013 varying)"). Data was extracted, downloaded, and analyzed using SEER*Stat Software 8.3.2. The patient's personal information was not exposed. The Ethics Committee of the Health Science Center of Xi' an Jiaotong University approved the study.

\section{Patient Selection Criteria}

From the SEER database, 19,010 patients with primary diagnosis 65 years of age were enrolled, and 12,407 patients were obtained according to the inclusion and exclusion criteria. ${ }^{9}$ Patients with unknown diagnosis year, age, gender, pathological type, pathological grade, tumor location, or tumor stage were excluded. Patients with unknown treatment status were excluded.

All patients were clinically staged according to the American Joint Committee on Cancer (AJCC) 6th edition. ${ }^{10}$ Surgery patients in this study referred to those who had esophagectomy. ${ }^{11}$ Patients who received local tumor destruction (Esophagus Surgery Code 10-14), local tumor excision (Esophagus Surgery Code 20-27), or unknown surgery techniques (Esophagus Surgery Code 90) were not considered to be surgerical patients.

For the purposes of this study, we obtained valid patients with a diagnosis year in between 2004 and 2013, pathological type of squamous cell carcinoma, and tumor stage of locally advanced stage (clinically staged as $\mathrm{T} 3-4 \mathrm{~N} 0 / \mathrm{xM} 0$ or T1$\left.4 \mathrm{~N} 1 \mathrm{M} 0^{10}\right)$.

\section{Survival Quantification}

Cancer-specific survival (CSS) was used to evaluate survival time. Survival data was extracted at 1-month intervals for a minimum follow-up of 4 months to exclude patients survived not long enough to receive cancer-directed therapy. CSS was determined from the SEER cause-specific death classification variable and was evaluated from the date of diagnosis to the date of EC specific death.

\section{Statistical Analysis}

Enrolled patients were first divided into five subgroups by treatment (Fig. 1). Patient's characteristics were compared by chi-square test. CSS was calculated using the KaplanMeier analysis and was compared by the log-rank test. Univariable and multivariable Cox proportional hazard models were established to identify covariates associtaed with CSS. We also used the Cox proportional hazards model to calculate HRs and 95\%CIs. Finally, stratified survival by age was delineated by Kaplan-Meier analysis. Significance was identified according to predefined threshold 0.05. All statistical analyses were implemented using the statistical analysis software SPSS 22.0 for Windows (SPSS, Inc., Chicago, IL, USA).

\section{Results}

\section{Patient Characteristics}

A total of 972 cases with primary diagnosis of locally advanced ESCC were enrolled. 251 (25.8\%) patients received surgery. Among the 972 patients, 331 cases (34.1\%) were age 65-69 years, 235 cases $(24.2 \%)$ were age $70-74$ years, 212 cases $(21.8 \%)$ were age $75-79$ years, and 194 cases $(20.0 \%)$ were age 80 years or older. The majority of patients had tumor diagnosed in cT3 $(60.2 \%)$, cN1 (66.7\%), and in the thoracic esophagus $(84.8 \%)$. The characteristics of the enrolled patients by treatment are shown in Table 1.

\section{Surgery and RT Utilization}

The percentage of patients receiving surgery alone has no significant difference across age groups. However, the percentage for patients receiving NRT + surgery therapy decreased over age (Table 1). Of patients, $20.2 \%$ were treated by NRT + surgery for the 65-69 years age group, while the percentage dropped to $18.3 \%$ and $5.7 \%$ for 70 74 years and age 75-79 years age groups, respectively. Only 3 out of 194 patients age $\geq 80$ years received NRT + surgery. The same decresing trend was also 
Fig. 1 Study workflow. NRT neoadjuvant radiotherapy, PORT postoperative radiotherapy, RT radiotherapy

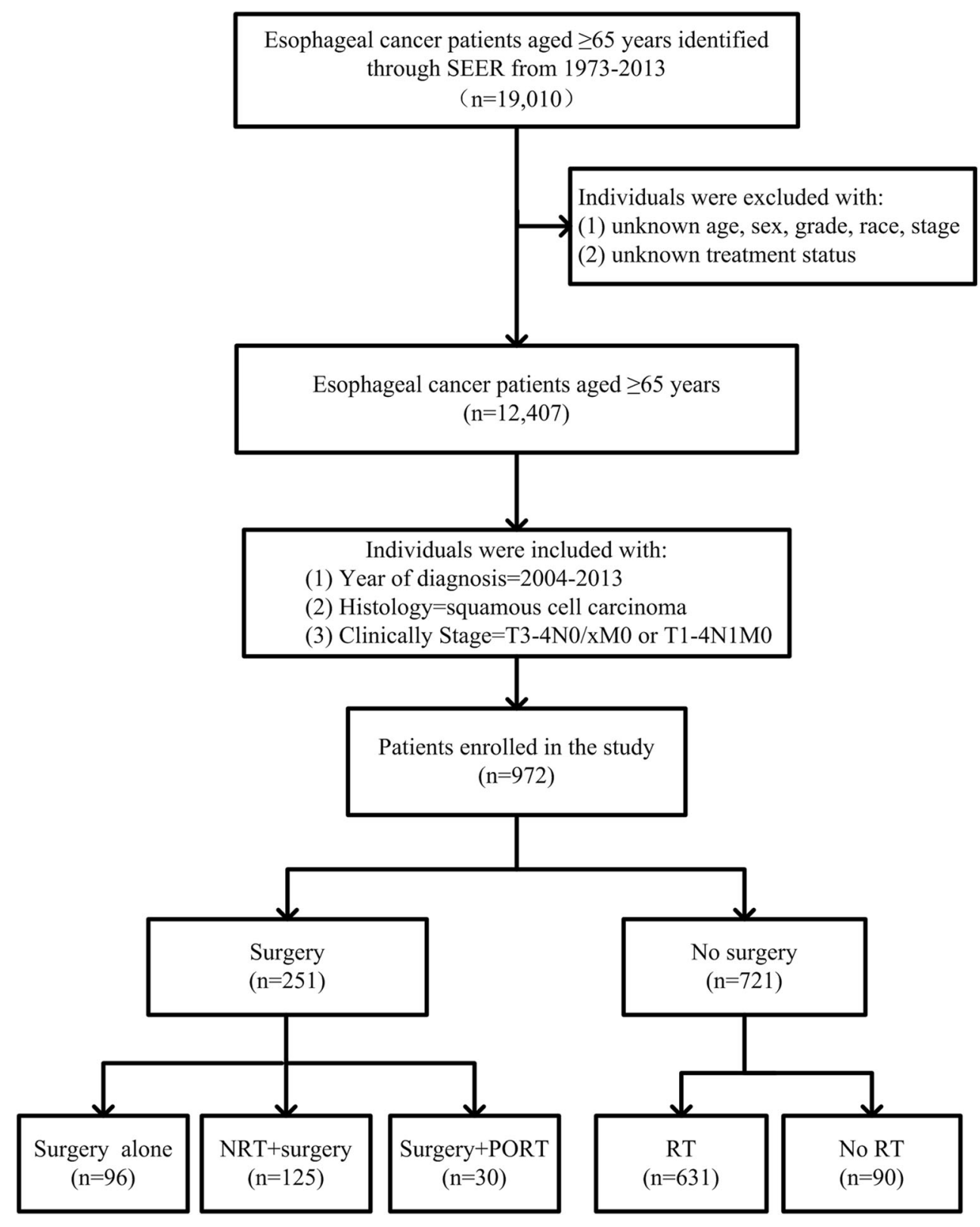

observed for percentage of patients receiving surgery + postoperative radiotherapy (PORT). Patients receving RT alone increased from $58.9 \%$ of $65-69$ years old to $72.7 \%$ of patients $\geq 80$ years old. For locally advanced ESCC aged 65-79 years, 553 patients did not receive surgery and most of them received RT $(88.6 \%)$.

For those aged 65-79 years who received surgery, 74 patients $(32.9 \%)$ underwent surgery alone, 122 patients $(54.2 \%)$ had received NRT followed by surgery, and 29 patients $(12.9 \%)$ had received surgery + PORT.

For patients aged over 80 , only 3 received NRT + surgery and one single patient received surgery + PORT. The limited number of such cases was not suciffient to draw conclusion for the group and combining them into other age groups may introduce additional bias. Hence, the patients aged over 80 were excluded in further analysis.

\section{Survival Benefit Evaluation}

We first compared the baseline conditions for three groups (surgery alone, NRT + surgery and surgery + PORT, Supplementary Table 1). A total of 225 patients aged 65-79 were included. Patients in the NRT + surgery groups were younger on average $(P<0.001)$ and more likely to have clinical positive lymph nodes $(P=0.002)$.

For the whole cohort of 65-79 years old, there was an improvement in CSS assoctiated with NRT + surgery (Table 3, Fig. 2). The median and 2-year CSS was 29.0 months 
Table 1 Characteristics of elderly patients with locally advanced ESCC, SEER 2004-2013

\begin{tabular}{|c|c|c|c|c|c|c|c|}
\hline \multirow[t]{2}{*}{ Characteristics } & \multicolumn{5}{|c|}{ No. $(\%)$ of patients } & \multirow[t]{2}{*}{$\chi^{2 *}$} & \multirow[t]{2}{*}{$P^{*}$} \\
\hline & $\begin{array}{l}\text { Surgery alone } \\
(n=96)\end{array}$ & $\begin{array}{l}\text { NRT + surgery } \\
(n=125)\end{array}$ & $\begin{array}{l}\text { Surgery + PORT } \\
(n=30)\end{array}$ & $\begin{array}{l}\text { RT alone } \\
(n=631)\end{array}$ & $\begin{array}{l}\text { No surgery or RT } \\
(n=90)\end{array}$ & & \\
\hline Age (years) & & & & & & 64.47 & $<0.001$ \\
\hline $65-69$ & $31(9.4 \%)$ & $67(20.2 \%)$ & $13(3.9 \%)$ & $195(58.9 \%)$ & $25(7.6 \%)$ & & \\
\hline $70-74$ & $21(8.9 \%)$ & $43(18.3 \%)$ & $9(3.8 \%)$ & $139(59.1 \%)$ & $23(9.8 \%)$ & & \\
\hline $75-79$ & $22(10.4 \%)$ & $12(5.7 \%)$ & $7(3.3 \%)$ & $156(73.6 \%)$ & $15(7.1 \%)$ & & \\
\hline $80-85+$ & $22(11.3 \%)$ & $3(1.5 \%)$ & $1(0.5 \%)$ & $141(72.7 \%)$ & $27(13.9 \%)$ & & \\
\hline Race & & & & & & 14.74 & 0.064 \\
\hline White & $71(10.2 \%)$ & $99(14.2 \%)$ & $18(2.6 \%)$ & $451(64.6 \%)$ & $59(8.5 \%)$ & & \\
\hline Black & $9(6.1 \%)$ & $10(6.8 \%)$ & $8(5.4 \%)$ & $102(68.9 \%)$ & $19(12.8 \%)$ & & \\
\hline Others & $16(12.7 \%)$ & $16(12.7 \%)$ & $4(3.2 \%)$ & $78(61.9 \%)$ & $12(9.5 \%)$ & & \\
\hline Sex & & & & & & 4.90 & 0.298 \\
\hline Female & $33(9.5 \%)$ & $43(12.4 \%)$ & $16(4.6 \%)$ & $219(63.3 \%)$ & $35(10.1 \%)$ & & \\
\hline Male & $63(10.1 \%)$ & $82(13.1 \%)$ & $14(2.2 \%)$ & $412(65.8 \%)$ & $55(8.8 \%)$ & & \\
\hline Grade & & & & & & 6.83 & 0.555 \\
\hline Well & $2(4.5 \%)$ & $2(4.5 \%)$ & $1(2.3 \%)$ & $33(75.0 \%)$ & $6(13.6 \%)$ & & \\
\hline Moderately & $44(10.0 \%)$ & $57(12.9 \%)$ & $16(3.6 \%)$ & $282(63.8 \%)$ & $43(9.7 \%)$ & & \\
\hline Poorly or Un & $50(10.3 \%)$ & $66(13.6 \%)$ & $13(2.7 \%)$ & $316(65.0 \%)$ & $41(8.4 \%)$ & & \\
\hline Tumor location & & & & & & 37.16 & 0.002 \\
\hline Cervical esophagus & $2(4.3 \%)$ & $1(2.2 \%)$ & $1(2.2 \%)$ & $40(87.0 \%)$ & $2(4.3 \%)$ & & \\
\hline $\begin{array}{l}\text { Thoracic } \\
\text { esophagus }\end{array}$ & $84(10.2 \%)$ & $115(14.0 \%)$ & $23(2.8 \%)$ & $525(63.7 \%)$ & $77(9.3 \%)$ & & \\
\hline $\begin{array}{l}\text { Abdominal } \\
\text { esophagus }\end{array}$ & $3(37.5 \%)$ & $2(25.0 \%)$ & $0(0.0 \%)$ & $3(37.5 \%)$ & $0(0.0 \%)$ & & \\
\hline Overlapping area & $5(11.1 \%)$ & $3(6.7 \%)$ & $5(11.1 \%)$ & $29(64.4 \%)$ & $3(6.7 \%)$ & & \\
\hline Location unknown & $2(4.1 \%)$ & $4(8.2 \%)$ & $1(2.0 \%)$ & $34(69.4 \%)$ & $8(16.3 \%)$ & & \\
\hline cT stage & & & & & & 58.72 & $<0.001$ \\
\hline $\mathrm{T} 1$ & $9(8.7 \%)$ & $7(6.8 \%)$ & $2(1.9 \%)$ & $73(70.9 \%)$ & $12(11.7 \%)$ & & \\
\hline $\mathrm{T} 2$ & $12(13.6 \%)$ & $17(19.3 \%)$ & $2(2.3 \%)$ & $51(58.0 \%)$ & $6(6.8 \%)$ & & \\
\hline $\mathrm{T} 3$ & $72(12.3 \%)$ & $89(15.2 \%)$ & $23(3.9 \%)$ & $361(61.7 \%)$ & $40(6.8 \%)$ & & \\
\hline $\mathrm{T} 4$ & $3(2.1 \%)$ & $9(6.3 \%)$ & $3(2.1 \%)$ & $106(73.6 \%)$ & $23(16.0 \%)$ & & \\
\hline $\mathrm{Tx}$ & $0(0.0 \%)$ & $3(5.8 \%)$ & $0(0.0 \%)$ & $40(76.9 \%)$ & $9(17.3 \%)$ & & \\
\hline $\mathrm{cN}$ stage & & & & & & 39.47 & $<0.001$ \\
\hline No & $38(12.4 \%)$ & $32(10.5 \%)$ & $17(5.6 \%)$ & $202(66.0 \%)$ & $17(5.6 \%)$ & & \\
\hline N1 & $57(8.8 \%)$ & $93(14.4 \%)$ & $13(2.0 \%)$ & $419(64.7 \%)$ & $66(10.2 \%)$ & & \\
\hline $\mathrm{Nx}$ & $1(5.6 \%)$ & $0(0.0 \%)$ & $0(0.0 \%)$ & $10(55.6 \%)$ & $7(38.9 \%)$ & & \\
\hline
\end{tabular}

ESCC esophageal squamous cell carcinoma, NRT neoadjuvant radiotherapy, PORT postoperative radiotherapy, $R T$ radiotherapy

*Chi-square test

and $54.4 \%$ for NRT + surgery and 17.0 months and $38.1 \%$ for surgery alone $(P=0.009)$ (Table 2$)$. However, there was no difference in CSS associated with PORT for the all whole cohort of 65-79 years old $(P=0.928)$. Multivariate analysis (Table 3 ) showed that after controlling the possible confounding factors, NRT + surgery remained associated with improved CSS (HR, 0.58; 95\%CI, 0.39 to $0.85 ; P=0.005$ ). However, for surgery + PORT group, we failed to observe significant survival benefit comparing to surgery alone group (HR, $0.98 ; 95 \%$ CI, 0.60 to $1.62 ; P=0.944$ ).

\section{Survival by Age}

As shown by Table 1, elder surgical patients were less likely to be treated by additional NRT, and we further conducted stratified survival analysis in different age groups. As shown by Fig. 3, the outcome of patients receiving additional NRT differed greatly across different aged group. For the locally advanced ESCC patients age 65-74, there was an CSS benefit of NRT compared with surgery alone, with the 2-year CSS being $56.6 \%$ for NRT + surgery and $39.6 \%$ for surgery alone $(P=$ 


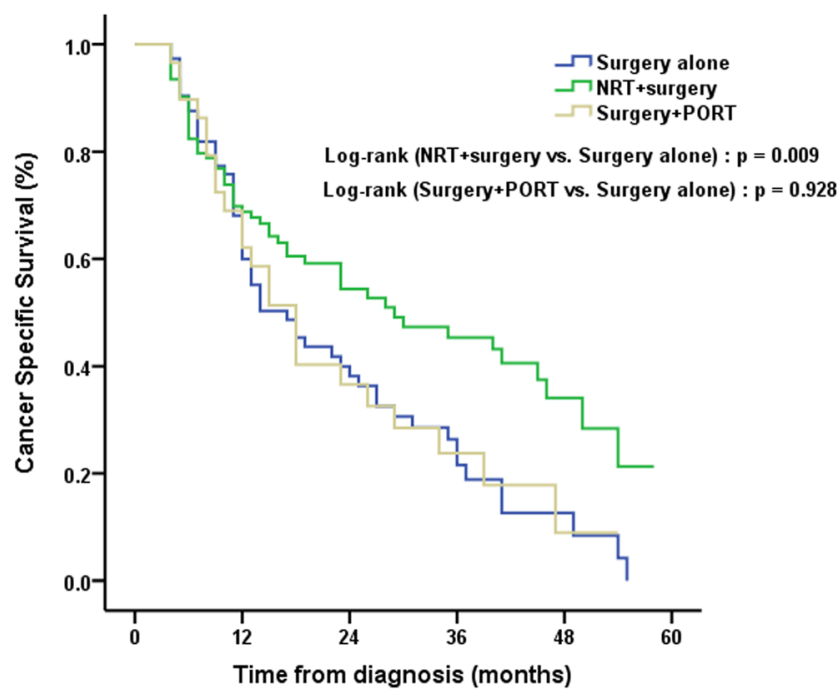

Fig. 2 Kaplan-Meier analysis for cancer-specific survival for locally advanced ESCC patients aged 65-79. ESCC esophageal squamous cell carcinoma, NRT neoadjuvant radiotherapy, PORT postoperative radiotherapy

0.026). However, for the patients aged 75-79 years, there were no difference in CSS $(P=0.972)$.

\section{Discussion}

Using large national cohort data from SEER database, we compared the CSS benefit from the addition of RT on esophagectomy for locally advanced ESCC patients aged $\geq 65$. In the study, compared with surgery alone, NRT significantly

Table 2 Patients survival stratified by age, SEER 2004-2013

\begin{tabular}{lllll}
\hline \multirow{2}{*}{ Cohort } & \multicolumn{2}{l}{ Cancer-specific survival } & & \\
\cline { 2 - 5 } & MST (months) & 2-year CSS (\%) & $\chi^{2 *}$ & $P^{*}$ \\
\hline Overall cohort & & & & \\
Surgery alone & 17.0 & 38.1 & Reference \\
NRT + surgery & 29.0 & 54.4 & 6.90 & 0.009 \\
Surgery + PORT & 18.0 & 36.6 & $<0.01$ & 0.928 \\
65-74 years old & & & & \\
Surgery alone & 18.0 & 39.6 & Reference \\
NRT + surgery & 30.0 & 56.6 & 4.93 & 0.026 \\
Surgery + PORT & 18.0 & 43.6 & 0.49 & 0.486 \\
75-79 years old & & & & \\
Surgery alone & 14.0 & 34.8 & Reference \\
NRT + surgery & 6.0 & 31.3 & $<0.01$ & 0.972 \\
Surgery + PORT & 8.0 & 14.3 & 2.99 & 0.084 \\
\hline
\end{tabular}

MST and 2-year survival rate were determined by Kaplan-Meier analysis $N R T$ neoadjuvant radiotherapy, PORT postoperative radiotherapy, $R T$ radiotherapy, MST median survival time, CSS cancer-specific survival

*Log-rank test increased CSS. On subgroup anlysis, the benefit was driven by patients aged 65 to 74 years.

Multiple studies have observed the clinical benefit of NRT to espaghal cancer patient survival. The CROSS trial indicated that patients with esophageal or esophagogastric junction cancer benefit from preoperative chemoradiotherapy, and many patients have complete pathological reaction. ${ }^{8}$ In addition, in locally advanced ESCC, NEOCRTEC5010 demonstrated that preoperative chemoradiotherapy + surgery improved survival compared with surgery alone. ${ }^{5}$ However, elder patients were not recruited in the trials. In the current study, we observed that in locally advanced ESCC patients aged over 65 , additional NRT treatment group has better outcome than PORT group or surgery alone group. This result was also observed in CROSS and NEOCRTEC5010 studies. Intriguingly, our analysis showed that there was an age-related differences in CSS benefit from NRT. Clinical benefit of NRT is only observed in patients aged between 65 and 74, not for those aged over 75. This has not been studied previously, since the number of patients receiving surgery alone was unknown in both CROSS and NEOCRTE5010 studies. We failed to observe the significant contribution of age in multiple Cox regression probably due to its dependence with other variable. Further studies with larger dataset and more advanced statistical modeling are warranted to confirm and interpret this finding.

Whether the treatment of PORT is beneficial to the overall survival of locally advanced ESCC is controversial. For patients who have undergone surgery, many studies failed to detect the survival benefit from the addition of PORT. ${ }^{12}$, ${ }^{13}$ One study by Xiao et al., however, reported that, for locally advanced ESCC, surgery with PORT can benefit patients with stage III and lymph node positive. ${ }^{14}$ In the current study, we evaluated the survival benefit of surgery plus PORT in an elderly cohort of patients with locally advanced ESCC. The result showed that comparing to surgery alone, additional PORT did not bring survival benefit to patients aged over 65 . Negative result was also observed in further age-stratified analysis. A possible explanation is that, in clinical practice, comparing to younger patient, a longer period is needed for an elderly patient to recover and receive PORT, which may reduce the treatment effect. In addition, elderly patients were also more vulnerable to additional PORT.

To our knowledge, this is the largest study to date specifically examing the role of surgery and RT for the elderly patients with locally advanced ESCC. However, our study has limitations. Firstly, the study is conducted in a retrospective, nonrandomized manner based on the SEER database and tend to be influenced by selection biases. In particular, many influencial factors, surgery type (i.e., if the surgery is a salvage resection), comorbidities, performance status, as well as patient 
Table 3 Cox proportional hazards analysis for cancer special survival of locally advanced ESCC patients aged 65-79, SEER 2004-2013

\begin{tabular}{|c|c|c|c|c|c|c|}
\hline \multirow[t]{2}{*}{ Variables } & \multicolumn{3}{|c|}{ Univariable analysis } & \multicolumn{3}{|c|}{ Multivariable analysis } \\
\hline & $P^{*}$ & $\mathrm{HR}^{*}$ & $95 \% \mathrm{CI}$ & $P$ & $\mathrm{HR}^{*}$ & $95 \% \mathrm{CI}$ \\
\hline Age (years) & 0.047 & & & 0.316 & & \\
\hline $65-74$ & Reference & 1 & & Reference & 1 & \\
\hline $75-79$ & 0.047 & 1.21 & $1.00-1.47$ & & 1.11 & $0.91-1.35$ \\
\hline Race & 0.622 & & & 0.469 & & \\
\hline White & Reference & 1 & & Reference & 1 & \\
\hline Black & 0.540 & 1.08 & $0.85-1.37$ & 0.792 & 1.03 & $0.81-1.32$ \\
\hline Others & 0.520 & 0.92 & $0.70-1.19$ & 0.256 & 0.86 & $0.65-1.12$ \\
\hline Sex & 0.598 & & & 0.679 & & \\
\hline Female & Reference & 1 & & Reference & 1 & \\
\hline Male & 0.598 & 1.05 & $0.87-1.26$ & 0.679 & 1.04 & $0.86-1.26$ \\
\hline Grade & 0.254 & & & 0.297 & & \\
\hline Well & Reference & 1 & & Reference & 1 & \\
\hline Moderately & 0.176 & 1.42 & $0.85-2.36$ & 0.158 & 1.45 & $0.87-2.42$ \\
\hline Poorly or Un & 0.348 & 1.27 & $0.77-2.11$ & 0.277 & 1.33 & $0.80-2.22$ \\
\hline Tumor location & 0.366 & & & 0.730 & & \\
\hline Cervical esophagus & Reference & 1 & & Reference & 1 & \\
\hline Thoracic esophagus & 0.254 & 0.80 & $0.55-1.17$ & 0.552 & 0.89 & $0.60-1.32$ \\
\hline Abdominal esophagus & 0.908 & 1.06 & $0.41-2.74$ & 0.722 & 1.19 & $0.45-3.15$ \\
\hline Overlapping area & 0.379 & 0.79 & $0.46-1.35$ & 0.483 & 0.82 & $0.47-1.42$ \\
\hline Location unknown & 0.755 & 1.08 & $0.66-1.79$ & 0.765 & 1.08 & $0.65-1.81$ \\
\hline cT stage & $<0.001$ & & & $<0.001$ & & \\
\hline $\mathrm{T} 1$ & Reference & 1 & & Reference & 1 & \\
\hline $\mathrm{T} 2$ & 0.322 & 1.23 & $0.82-1.85$ & 0.193 & 1.32 & $0.87-2.00$ \\
\hline $\mathrm{T} 3$ & 0.344 & 1.16 & $0.86-1.56$ & 0.165 & 1.25 & $0.91-1.72$ \\
\hline $\mathrm{T} 4$ & 0.000 & 2.24 & $1.59-3.15$ & 0.000 & 2.33 & $1.62-3.33$ \\
\hline $\mathrm{Tx}$ & 0.010 & 1.83 & $1.15-2.92$ & 0.024 & 1.72 & $1.07-2.77$ \\
\hline cN stage & 0.465 & & & 0.675 & & \\
\hline No & Reference & 1 & & Reference & 1 & \\
\hline $\mathrm{N} 1$ & 0.708 & 1.04 & $0.86-1.26$ & 0.395 & 1.10 & $0.88-1.37$ \\
\hline $\mathrm{Nx}$ & 0.219 & 1.53 & $0.78-2.99$ & 0.678 & 1.16 & $0.58-2.31$ \\
\hline Treatment & $<0.001$ & & & $<0.001$ & & \\
\hline Surgery alone & Reference & 1 & & Reference & 1 & \\
\hline NRT + surgery & 0.012 & 0.62 & $0.43-0.90$ & 0.005 & 0.58 & $0.39-0.85$ \\
\hline Surgery + PORT & 0.910 & 0.97 & $0.60-1.58$ & 0.944 & 0.98 & $0.60-1.62$ \\
\hline RT alone & 0.898 & 1.02 & $0.76-1.36$ & 0.431 & 0.89 & $0.66-1.20$ \\
\hline No surgery or RT & 0.004 & 1.80 & $1.21-2.66$ & 0.039 & 1.55 & $1.02-2.33$ \\
\hline
\end{tabular}

ESCC esophageal squamous cell carcinoma, NRT neoadjuvant radiotherapy, PORT postoperative radiotherapy, $R T$ radiotherapy, $H R$ hazard ratio, $C I$ confidence interval

*HR and $P$ values were derived from Cox proportional hazards regression analysis, and multivariable models were adjusted for all confounding factors listed in the table choice, were not recorded in SEER. The possible imbalanced distribution across groups may bias the result. Secondly, to our knowledge, this study is currently the largest study on elderly locally advanced ESCC patients, still the sample sizes for subgroups, especially for group aged over 80 , were quite small. We previously have conducted a retrospective populationbased study for EC patients aged over 80 to evaluate the survival benefit of RT, observing that patients benefits from RT only in localized/regional stage. ${ }^{9}$ However, in the current study, the distribution of patients aged over 80 was very different comparing to treatment groups, with very limited number of cases in comined therapy groups and therefore was removed to avoid additional analysis bias. Additionally, the SEER database contains only a binary variable for chemical 

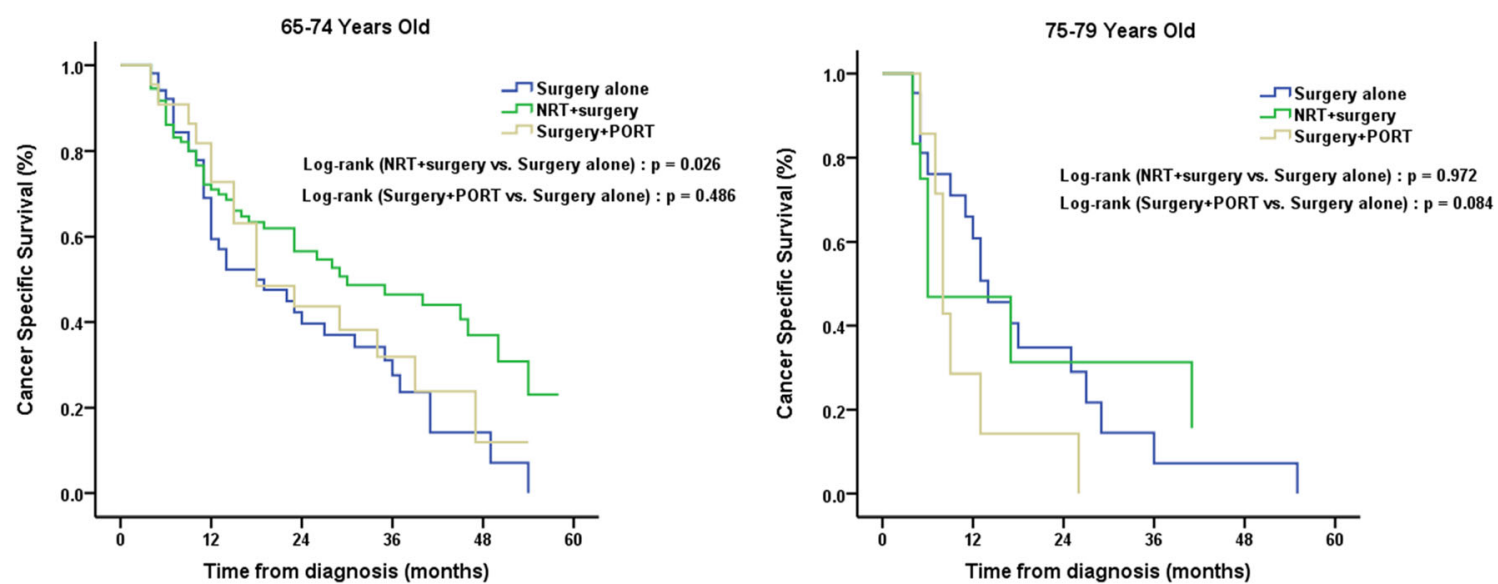

Fig. 3 Kaplan-Meier analysis for cancer-specific survival for locally advanced ESCC patients stratified by age. ESCC esophageal squamous cell carcinoma, NRT neoadjuvant radiotherapy, PORT postoperative radiotherapy

treatment. To our knowledge, comparing to RT, the effect of chemotherapy varies much greater for different treatment plan. ${ }^{15,16}$ Using only the binary information without dose and period information may introduce new biaes and was not included in the current study. Despite above mentioned limits, the existing data did suggest that it is worthy of extending current effort with a prospective study in identifying the most appropriate treatment for the patients of different age subgroups.

\section{Conclusion}

For the elderly patients with locally advanced ESCC, this SEER-based study suggested an overall benefit from NRT over surgery alone in patients younger than age 65-74 years but not in patients age 75-79 years. Further, well-designed prospective studies are required to more fully address this issue.

Author Contributions Conception and design: Shan Huang.

Development of methodology: Shan Huang, Yuxing Li.

Acquisition of data: Hongbing Ma, Shuyu Zheng, Yue Ke.

Analysis and interpretation of data: Wenyu Wang, Fang Li.

Writing, review and/or revision of the manuscript: Shan Huang,

Wenyu Wang.

Study supervision: Shan Huang.

All authors have revised the manuscript and approved the final version to be published.

Funding This work was supported by the Natural Science Foundation of Shaanxi Province [grant number 2017JM8099] and the Key Project of the Second Affiliated Hospital, Xi'an Jiaotong University [grant number YJ(ZD)201705].

\section{Compliance with Ethical Standards}

The Ethics Committee of the Health Science Center of Xi'an Jiaotong University approved the study.
Conflict of Interest The authors declare that they have no conflicts of interest.

\section{References}

1. Siegel RL, Miller KD, Jemal A. Cancer Statistics, 2017. CA Cancer J Clin. 2017; 67: 7-30.

2. Pennathur A, Gibson MK, Jobe BA, Luketich JD. Oesophageal carcinoma. Lancet. 2013; 381: 400-412.

3. Howlader N, Noone A, Krapcho M. SEER cancer statistics review, 1975-2013. December 17, 2014. National Cancer Institute. 2017.

4. Daly JM, Fry WA, Little AG, Winchester DP, McKee RF, Stewart AK, Fremgen AM. Esophageal cancer: results of an American College of Surgeons Patient Care Evaluation Study. J Am Coll Surg. 2000; 190: 562-572; discussion 572-563.

5. Yang H, Liu H, Chen Y, Zhu C, Fang W, Yu Z, Mao W, Xiang J, Han Y, Chen Z, Yang H, Wang J, Pang Q, et al. Neoadjuvant Chemoradiotherapy Followed by Surgery Versus Surgery Alone for Locally Advanced Squamous Cell Carcinoma of the Esophagus (NEOCRTEC5010): A Phase III Multicenter, Randomized, Open-Label Clinical Trial. J Clin Oncol. 2018; 36: 2796-2803.

6. Mariette C, Dahan L, Mornex F, Maillard E, Thomas PA, Meunier B, Boige V, Pezet D, Robb WB, Le Brun-Ly V, Bosset JF, Mabrut JY, Triboulet JP, et al. Surgery alone versus chemoradiotherapy followed by surgery for stage I and II esophageal cancer: final analysis of randomized controlled phase III trial FFCD 9901. J Clin Oncol. 2014; 32: 2416-2422.

7. Mak RH, Mamon HJ, Ryan DP, Miyamoto DT, Ancukiewicz M, Kobayashi WK, Willett CG, Choi NC, Blaszkowsky LS, Hong TS. Toxicity and outcomes after chemoradiation for esophageal cancer in patients age 75 or older. Dis Esophagus. 2010; 23: 316-323.

8. van Hagen P, Hulshof MC, van Lanschot JJ, Steyerberg EW, van Berge Henegouwen MI, Wijnhoven BP, Richel DJ, Nieuwenhuijzen GA, Hospers GA, Bonenkamp JJ, Cuesta MA, Blaisse RJ, Busch $\mathrm{OR}$, et al. Preoperative chemoradiotherapy for esophageal or junctional cancer. N Engl J Med. 2012; 366: 2074-2084.

9. Huang S, Zheng S, Gong T, Ma H, Ke Y, Zhao S, Wang W, Jia L, Zhang X. Survival benefit evaluation of radiotherapy in esophageal cancer patients aged 80 and older. Oncotarget. 2017; 8: 112094 112102.

10. Greene FL, Page DL, Fleming ID (eds): AJCC Cancer Staging Manual (ed 6). New York, NY, Springer-Verlag, 2002. 
11. National Cancer Institute. Appendix C: Site Specific Surgery Codes. https://seer.cancer.gov/manuals/2018/AppendixC/Surgery Codes_Esophagus_2018.pdf.

12. Teniere P, Hay JM, Fingerhut A, Fagniez PL. Postoperative radiation therapy does not increase survival after curative resection for squamous cell carcinoma of the middle and lower esophagus as shown by a multicenter controlled trial. French University Association for Surgical Research. Surg Gynecol Obstet. 1991; 173: $123-130$.

13. Fok M, Sham JS, Choy D, Cheng SW, Wong J. Postoperative radiotherapy for carcinoma of the esophagus: a prospective, randomized controlled study. Surgery. 1993; 113: 138-147.

14. Xiao ZF, Yang ZY, Liang J, Miao YJ, Wang M, Yin WB, Gu XZ, Zhang DC, Zhang RG, Wang LJ. Value of radiotherapy after radical surgery for esophageal carcinoma: a report of 495 patients. Ann Thorac Surg. 2003; 75: 331-336.
15. Zheng Y, Li Y, Liu X, Sun H, Wang Z, Zhang R. Reevaluation of Neoadjuvant Chemotherapy for Esophageal Squamous Cell Carcinoma: A Meta-Analysis of Randomized Controlled Trials Over the Past 20 Years. Medicine (Baltimore). 2015; 94: e1102.

16. Sjoquist KM, Burmeister BH, Smithers BM, Zalcberg JR, Simes RJ, Barbour A, Gebski V, Australasian Gastro-Intestinal Trials G. Survival after neoadjuvant chemotherapy or chemoradiotherapy for resectable oesophageal carcinoma: an updated meta-analysis. Lancet Oncol. 2011; 12: 681-692.?show "reftot"\$x(^refent)>

Publisher's Note Springer Nature remains neutral with regard to jurisdictional claims in published maps and institutional affiliations. 